\title{
8 CONCLUSIONES, RECOMENDACIONES, LÍMITES Y FUTURAS INVESTIGACIONES
}

\subsection{CONCLUSIONES DEL TRABAJO}

El presente trabajo planteó como objetivo genérico el estudio del Sistema de Innovación en el Sector Sanitario Público Catalán - por lo tanto, en éste trabajo no se estudió el sector sanitario privado catalán que representa alrededor de un $20 \%$ del total del sector. Tampoco se estudió en este trabajo los consorcios participados por el SCS, o las relaciones que el SSPC pueda tener con otros sectores del cluster de la salud o con los demás sectores de la economía catalana- a través del análisis de la estructura propuesta por la Llei d'Ordenació Sanitària de Catalunya. Para cubrir este objetivo el trabajo se dividió en dos partes.

En la primera parte, que abarca los capítulos primero y segundo se revisaron diversos enfoques conceptuales con el fin de describir un marco teórico que ayude a explicar el Sistema de Innovación. En ésta parte se pretendieron dos objetivos: en primer lugar, analizar las aportaciones conceptuales explicativas del fenómeno de la Innovación y en segundo lugar, analizar las diversas perspectivas teóricas sobre el Sistema Nacional de Innovación, las conclusiones se encuentran al final de cada unos de los capítulos.

En la segunda parte, en los capítulos tercero, cuarto, quinto, sexto y séptimo se concentraron los objetivos específicos del trabajo y su contrastación empírica. Con relación al capítulo cuarto, las conclusiones se encuentran al final del capítulo.

Es conveniente ahora sistematizar las conclusiones de los capítulos quinto, sexto y séptimo. 
(1) Respecto a la historia hospitalaria catalana. Del estudio hecho se derivan, según nuestro punto de vista, las siguientes conclusiones:

Históricamente, y más significativamente desde principio de siglo, la sociedad civil catalana demostró con sus iniciativas a través del desarrollo de un conjunto de centros y de servicios sanitarios nacidos de la iniciativa de diversas entidades benéficas asistenciales, de las corporaciones locales, de las mutualidades, de la Iglesia y de las sociedades cooperativas que organizaron una sólida red de hospitales comarcales en un conjunto de instituciones públicas y privadas.

Un testimonio evidente es la Figura 5.1 que muestra las Ordenaciones del Hospital de la Santa Creu i Sant Pau en el año 1707. Otro testimonio es la Figura 5.2 que publica la Conselleria de Sanitat en el año 1933.

En esta época en Catalunya ya había un plan de regionalización sanitaria basado en la estructura comarcal, uno de los puntos de diferenciación de la sociedad catalana.

Nuestro trabajo se situó en esta revisión de la historia y en esta explicación anterior porque pensamos que el modelo actual de la sanidad catalana está estrechamente vinculado con el pasado y el MS—80, el DMS—83, el PRH, la XHUP, etc. no habrían sido posibles sin esta historia protagonizada por la sociedad civil catalana.

(2) Respecto a la LOSC. Del estudio hecho se derivan, según nuestro punto de vista, las siguientes conclusiones:

Cuando en 1981 la Generalitat recibió las primeras trasferencias sanitarias, las camas de enfermos graves correspondientes a la Seguridad Social sólo representaban un 30\% de todas la oferta de camas en Catalunya. Esto significaba que había 0,77 camas de titularidad pública por mil habitantes, mientras que en el resto del Estado la proporción era de 1,91 camas por mil habitantes.

La autonomía en la gestión de servicios comienza con la elaboración del Mapa Sanitario de Catalunya [1981], que permite conocer con detalle la realidad sanitaria y constituye el primer instrumento de planificación. Las conclusiones de este primer periodo plantean la necesidad de definir un sistema sanitario que aproveche todas las infraestructuras existentes, mediante contratos y conciertos con entidades públicas y privadas, con el objetivo de complementar los recursos sanitarios y asistenciales públicos.

En 1981 se aprobó la orden de acreditación, que establecía los requisitos de estructura y de funcionamiento que debían cumplir los centros sanitarios que firmaban un concierto con el DSSS para la prestación de asistencia sanitaria pública. 
En 1983 se crea el ICS como entidad gestora de los centros y servicios de la Seguridad Social en Catalunya.

En 1985 se creala XHUP, integrada por todos los centros-independientemente de su titularidad jurídica- que prestan asistencia sanitaria a los titulares y beneficiarios de la Seguridad Social. También en 1985 se inicia la reforma de la atención primaria, que pone en funcionamiento los EAP, estos equipos también realizan tareas de prevención y de educación sanitaria en sus ABSs.

La existencia de la realidad histórica basada en un sistema mixto y el cambio cultural que se introdujo durante los años ochenta concluyen con una clara definición estratégica: considerar a la administración sanitaria como financiadora y compradora de servicios, disociándola de su papel de gestora directa de la producción.

Como paso intermedio se creó en 1989 la Dirección General de Recursos Económicos del Sistema Sanitario, responsable de la compra de los servicios sanitarios de provisión pública.

En 1990 se aprobó la LOSC, los rasgos característicos de la LOSC se concretan en los siguientes puntos:

- Se crea el SCS como ente público responsable de la prestación de los servicios sanitarios de cobertura pública de Catalunya.

- La atención integral de la salud, que incluye la promoción, prevención, curación y rehabilitación, es un derecho universal exigible para todos los ciudadanos residentes en Catalunya.

- Se potencia una descentralización de los servicios sanitarios que permita superar las desigualdades sociales y territoriales.

- Se configura un mercado de competencia planificada y regulada, mediante la separación de las funciones de financiación y de provisión de servicios y potenciando un proceso de diversificación de proveedores.

- La provisión de los servicios requiere un sistema sanitario mixto, tradicional en Catalunya, que permitió aprovechar todos los recursos existentes, ya sean públicos o privados.

- La Administración y la organización sanitaria deben tender a la racionalización, la eficacia, la simplificación y la eficiencia.

- Se debe promover la diversidad de fórmulas de gestión — directa, indirecta o compartida - y se debe abrir a los profesionales de la atención primaria la posibilidad de gestión de los centros.

- Los centros del sistema sanitario público se deben orientar hacia una concepción de empresas productivas de servicios, definiendo 
unos objetivos que permitan el control por resultados y con una gestión empresarial.

La modificación parcial de la LOSC se produjo básicamente por la obligada adecuación del régimen de contratación del SCS a la legislación básica del Estado y por la necesidad de regular con más precisión algunos aspectos como el procedimiento a seguir para la constitución de organismos que dependen del SCS, la formación de consorcios o las normas ordenadas para el establecimiento de fórmulas destinadas a la gestión de servicios sanitarios que se fundamentan en entidades de base asociativa de profesionales sanitarios.

No obstante, a partir del análisis, de las encuestas y de las entrevistas realizadas a lo largo del estudio del SI-SSPC podemos concluir que:

El SCS como ente financiador-asegurador único de la sanidad pública catalana participa en la provisión de los servicios sanitarios a través de los consorcios, empresas públicas y participa en los patronatos o juntas directivas de los hospitales de la XHUP. Por lo tanto, unos de los principios básicos de la LOSC de separación de las funciones de planificación y compra de las de provisión de servicios sanitarios no se cumplen, en otras palabras el SCS es al mismo tiempo financiador-proveedor de los servicios sanitarios en el SSPC.

El IES es un organismo autónomo y tendría que considerarse como instrumento de soporte al DSSS, del SCS y del ICS en materia de formación de los profesionales sanitarios y no-sanitarios del campo de la salud y coordinar y potenciar la investigación en ciencias de la salud —artículos 69, 70 y 71 de la LOSC—, está considerado por los demás actores como órgano certificador de las actividades científicas realizadas por éstos para poder recibir las subvenciones correspondientes que sin esta certificación no son liberadas por el DSSS.

También el IES está traspasando a otros agentes las funciones de formación de los profesionales sanitarios y no-sanitarios del sistema.

Con relación al Pla de serveis que pretende ser la cartera de servicios cubiertos por el financiador — asegurado—evaluador único—SCS— y que regulará todos los contratos de prestación de servicios de los proveedores con el SCS, todavía no ha sido implantado.

La Enquesta de Salut de Catalunya -ESCA—, que es uno de los puntos clave del Pla de salut y por consiguiente de la LOSC, pues es una de las principales herramientas de detección de las necesidades, definición de los objetivos y establecimiento de las prioridades para conseguir a través de actividades e intervenciones sanitarias los objetivos de salud propuestos por el Pla de salut, fue hecha por primera y última vez en 1994. 
(3) Respecto al entorno económico. Del estudio hecho se derivan, según nuestro punto de vista, las siguientes conclusiones:

Para la financiación de los servicios sanitarios, la Generalitat recibe de los recursos procedentes de la participación de Catalunya en el presupuesto estatal del Insalud, del propio sistema de financiación general de las comunidades autónomas y de las diputaciones que transfirieron servicios sanitarios a la Generalitat.

El DSSS transfiere al SCS los importes para financiar la provisión de servicios sanitarios públicos que prestan los centros de la red sanitaria de utilización pública, ya sean concertados o del ICS.

El SCS dedica su presupuesto mayoritariamente a los siguientes grupos de gasto: la concertación de servicios sanitarios con entidades proveedoras — 39\%—, la financiación de los servicios del ICS - 34\%—, el gasto en prescripción farmacéutica — 21\% - y los gasto de aportaciones de capital - 2\%-.

No obstante, a partir del análisis, de las encuestas y de las entrevistas realizadas a lo largo del estudio del SI-SSPC podemos concluir que:

El sector sanitario español y catalán están altamente politizados y legislatura tras legislatura sirven como moneda de cambio en los pactos parlamentarios de los distintos gobiernos.

El último modelo de financiación sanitaria pactado para el periodo 1998-2001 previene que Catalunya tendrá una ganancia del 5,71\% sobre el modelo anterior.

(4) Respecto al Pla de salut de Catalunya. Del estudio hecho se derivan, según nuestro punto de vista, las siguientes conclusiones:

Las necesidades de salud de los ciudadanos se detectan a través del PSC, que se configura como el instrumento fundamental de planificación sanitaria de la Generalitat de Catalunya. El PSC permitió introducir un cambio importante de orientación al incidir en la planificación por objetivos de salud por encima de una actuación según los recursos disponibles.

El PSC, que se actualiza cada tres años y que se evalúa anualmente, incorpora los objetivos de salud para todo el mundo para el año 2000 de la Organización Mundial de la Salud y se elabora con la participación de equipos multidisciplinarios integrados por profesionales de la salud, gestores sanitarios, representantes de la Administración y de las instituciones sanitarias, y con la participación comunitaria.

Para conocer con más fiabilidad las necesidades de salud específicas de cada grupo de población y para hacer más eficaces las intervenciones programadas, los trabajos se inician a partir de las regiones y de los sectores sanitarios, los órganos más cercanos a la población. 
Las principales líneas de intervención del PSC son las siguientes:

- Importancia de las actividades preventivas.

- Profundización en la información y educación sanitarias.

- Promoción de los hábitos saludables.

- Vigilancia epidemiológica.

- Mejoría en la coordinación entre los diferentes niveles asistenciales.

- Consenso clínico para abordar los problemas de salud.

- Promoción de la formación continuada y de la investigación de los profesionales.

- Importancia de la evaluación.

- Aumento de satisfacción de los usuarios a través de la mejoría de los servicios y de la facilidad para acceder a ellos.

No obstante, a partir del análisis, de las encuestas y de las entrevistas realizadas a lo largo del estudio del SI-SSPC podemos concluir que:

El Pla de salut de Catalunya es considerado por muchos actores como un documento de estantería y herramienta de marketing para la Generalitat. El periodo trienal de vigencia del plan es muy pequeño, no se puede plantear objetivos de salud a cada tres años.

Son demasiados los 263 objetivos de salud propuestos por el plan 19931995 y carecen de una metodología para su inclusión en el plan.

Los objetivos de salud del plan 1996-1998 son de continuidad del anterior plan y en el plan que está por ser presentado los objetivos siguen siendo de continuidad del primer. Objetivos éstos que la OMS había planteado como los de «salud para todos en el año 2000».

El plan de salud no elaboró ningún documento con los costes de cada uno de los objetivos de salud, tampoco creó el calendario y sistematización de las herramientas para la evaluación de esos objetivos incluyendo también el coste económico de esas acciones.

El plan no involucra a los actores del sistema en la investigación científica para la consecución de los objetivos de salud propuestos.

(5) Respecto al SCS. Del estudio hecho se derivan, según nuestro punto de vista, las siguientes conclusiones:

El sistema sanitario de Catalunya se organiza alrededor del SCS, creado como ente público de carácter institucional.

Para garantizar la prestación de los servicios sanitarios a los ciudadanos, el SCS está constituido por todos los recursos sanitarios públicos y de cobertura 
pública, que forman la red sanitaria de utilización pública de Catalunya. Para cumplir esta misión y para velar por la equidad, la calidad y la eficiencia de los servicios, el SCS pone al servicio de la red sanitaria su organización corporativa.

La organización corporativa del SCS se constituye en tres niveles: los servicios matrices y la estructura territorial, las empresas públicas y los órganos colegiales de dirección y participación comunitaria.

Para acercar los servicios sanitarios a la población y conseguir una mayor equidad en el acceso al sistema sanitario, el SCS fue estructurado de forma descentralizada a través de las regiones sanitarias, los sectores sanitarios y las áreas básicas de la salud.

Catalunya queda dividida en ocho regiones sanitarias delimitadas a partir de factores geográficos, socioeconómicos y demográficos, teniendo en cuenta la ordenación comarcal del territorio. Las regiones sanitarias despliegan su actividad en los ámbitos de la planificación, de la compra de servicios, de los sistemas de gestión y de la atención al cliente.

Las regiones sanitarias cuentan con una dotación de recursos sanitarios de atención primaria y de atención especializada y hospitalaria para atender las necesidades de la población. Cada región se ordena, a su vez, en sectores sanitarios.

Los sectores sanitarios son el ámbito donde se desarrollan y coordinan las actividades de promoción de la salud, prevención de la enfermedad, salud pública, asistencia sociosanitaria en el nivel de atención primaria y de las especialidades médicas. Los sectores sanitarios están constituidos por la agrupación de áreas básicas de salud, unas unidades territoriales formadas por barrios o distritos en las áreas urbanas y por uno o más municipios en el ámbito rural, con una población entre 5.000 y 25.000 habitantes, que pueden llegar a 40.000 en las ciudades. Actualmente existen 338 áreas básicas de salud.

La red sanitaria de utilización pública ofrece diferentes servicios esenciales: la atención primaria, la atención hospitalaria, la atención sociosanitaria, la atención psiquiátrica y la atención farmacéutica, juntamente con otros recursos más específicos y con un conjunto de prestaciones complementarias.

Atención primaria. Con más de 300 centros de atención primaria - CAP- y más de 650 consultorios locales, la atención primaria es el nivel asistencial básico, el más cercano a la población y, por tanto, el más utilizado. La atención primaria se encuentra inmersa en un profundo proceso de reforma, con más dedicación de los profesionales a la atención a los usuarios y con actuaciones de prevención y promoción de la salud. Esta reforma gira en torno a la implantación en las áreas básicas de salud de los equipos de atención primaria, integrados por profesionales 
sanitarios y no sanitarios. La atención primaria de la salud cubre los servicios de medicina general, de pediatría, la atención de enfermería y las urgencias, tanto en régimen ambulatorio como a domicilio. Progresivamente se están incorporando profesionales en odontología y en asistencia social. La atención especializada se puede prestar en las consultas de especialidades médicas de los centros de atención primaria o bien en los centros hospitalarios que están integrados en esta actividad.

Atención hospitalaria. Los hospitales de Catalunya que contratan la prestación de servicios con el SCS configuran la red hospitalaria de utilización pública - XHUP_. Los centros hospitalarios de esta red fueron clasificados en cuatro grupos: hospital general básico y hospital aislado, hospital general básico, hospital de referencia y hospital de alta tecnología. Los hospitales generales básicos tienen la tecnología asistencial necesaria para atender aquellas patologías que no requieren un grado de especialización importante ni un equipamiento sofisticado. Se considera que cubren entre un setenta y un ochenta por ciento de la demanda que genera la atención hospitalaria. Los hospitales de referencia dan respuesta a casi todos los problemas de salud, excepto aquellos casos de baja prevalencia que requieren recursos tecnológicos de alto nivel. Los hospitales de alta tecnología disponen de las técnicas diagnosticas y terapéuticas más modernas y atienden a los pacientes que no pueden ser tratados en los hospitales de referencia.

Atención sociosanitaria. Los recursos sociosanitarios se destinan a la atención de personas con enfermedades crónicas invalidantes o enfermedades terminales y a la asistencia a personas mayores con alguna enfermedad. El SCS cuenta con casi 5.000 plazas de internamiento para pacientes de larga estancia, convalecientes, para curas paliativas y para enfermos de SIDA. Además hay unas 500 plazas de hospitalización de día concertadas y funcionan equipos de atención domiciliaria y unidades interdisciplinarias de atención especial — geriátricas, de curas paliativas, de demencias, para el aparato respiratorio—. Son los PADES — programas de atención domiciliaria, equipos de apoyo-y las UFISS —unidades funcionales interdisciplinarias sociosanitarias-.

Atención psiquiátrica y salud mental. Los diversos centros y servicios que constituyen la red de atención psiquiátrica y salud mental se estructuran en tres líneas básicas: apoyo a la atención primaria, atención hospitalaria especializada y centros de rehabilitación — centros de día y pisos asistidos- En total son más de 4.500 camas y más de 1.000 plazas en centros y hospitales de día.

Otros recursos de atención sanitaria. Los centros de atención a las drogodependencias, los centros de planificación familiar, los bancos de sangre, los centros de trasplantes, los programas de promoción de la salud y de prevención 
de enfermedades, los traslados sanitarios, las prestaciones complementarias y las oficinas de atención farmacéutica son servicios que completan la red sanitaria pública de Catalunya.

A estos servicios se añaden otras prestaciones asistenciales. Se trata, entre otros, de los servicios de diagnóstico por la imagen, los laboratorios de análisis clínicos, los laboratorios de medicina nuclear, los servicios de rehabilitación, la oxigenoterapia y los tratamientos respiratorios a domicilio, los servicios de litotricia y los servicios de diálisis.

No obstante, a partir del análisis, de las encuestas y de las entrevistas realizadas a lo largo del estudio del SI-SSPC podemos concluir que:

El SCS como ya hablamos anteriormente tiene las funciones de planificadorcomprador-evaluador y proveedor de servicios sanitarios en el SSPC.

El SCS no fomenta la cooperación entre los actores del SSPC, en nuestro entender en el futuro del SCS deberá promover la cooperación y castigar la nocooperación entre los actores del SSPC como medio para mejorar la eficiencia y eficacia del mismo e intentar reducir costes con la horizontalización de las estructuras sanitarias.

El SCS debería haber concluido la Reforma de la Atención Primaria en 1996 y todavía hoy no está concluida.

El SCS no mantiene una línea de comunicación directa con cada usuario del sistema.

El SCS creó la Central de Balanços con la finalidad de prestar un servicio de soporte a los centros sanitarios del SCS y que consiste en la realización de informes de análisis comparativa económico-financiera de los centros a partir de información facilitada por los propios centros. El objetivo principal de la CB es facilitar la toma de decisiones de los centros sanitarios a partir del conocimiento de la situación del sector. Pero toda esa información conjunta del sector es totalmente privilegiada y hasta hoy no se hizo público ningún informe de la CB.

Algunos actores en un primer momento tuvieron recelos con la $\mathrm{CB}$ por no tener claro su finalidad, a partir de esa confidencialidad de la información que garantizó el SCS ya existe un movimiento reivindicativo de una Central de Resultados que vaya más allá del análisis puramente económico-financiero y proporcione una información más completa del sector, también reivindicamos que se haga público un resumen o toda la información de la CB para que la sociedad pueda tener acceso a esa información privilegiada, en nuestro caso para estudiarla. 
El SCS no posee una red de información homogénea implantada entre los centros sanitarios públicos y concertados.

La implantación de la tarjeta de identificación magnética-TIS Tarjeta individual sanitaria — todavía no es una realidad, en algunas Regiones sanitarias la cifra no alcanza el 70\%, imposibilitando la creación de la base de datos de los usuarios asegurados por el SCS.

(6) Respecto al ICS. Del estudio hecho se derivan, según nuestro punto de vista, las siguientes conclusiones:

El ICS es la entidad que gestiona directamente los centros sanitarios de titularidad pública, es decir, aquellos centros propios de la Seguridad Social, en algunos casos, centros propios de la Generalitat de Catalunya.

Actualmente cuenta con 11 hospitales, cerca de 300 centros de atención primaria y más de 150 servicios de atención primaria-centros de atención especializada, centros de rehabilitación, programas de atención a la mujer, atención domiciliaria, etc.- -

No obstante, a partir del análisis, de las encuestas y de las entrevistas realizadas a lo largo del estudio del SI-SSPC podemos concluir que:

El ICS está pasando por en proceso de transformación y de redefinición de su papel como gran proveedor público de servicios sanitarios dentro del SSPC.

El ICS encuentra el SCS como comprador y competencia en la provisión de servicios sanitarios, está duplicidad de papel que asume el SCS no es bien vista por algunos de los actores del sistema.

El ICS tampoco fomenta la relación de cooperación entre sus hospitales del Grupo 4. También en el futuro el ICS deberá promover la cooperación y castigar la no-cooperación entre los hospitales como medio para mejorar la eficiencia y eficacia de los mismos e intentar reducir costes con la horizontalización de las estructuras hospitalarias de alta tecnología.

(7) Respecto a las Empresas Públicas del SCS. Del estudio hecho se derivan, según nuestro punto de vista, las siguientes conclusiones:

Los cambios definidos para el sistema sanitario de Catalunya — separando la financiación y la provisión - exigen, tanto para los proveedores de servicios como para el SCS, la adopción de un nuevo modelo de funcionamiento más de acuerdo con el modelo empresarial y que supere el sistema tradicional de la Administración Pública. Este cambio se produjo tanto en la dirección estratégica como en la gestión económica. 
En un mercado de competencia planificada, los centros proveedores están obligados a ser eficientes para poder ofrecer la mejor relación calidad—coste, ya que en un proceso de diversificación de proveedores y en una época de limitación de recursos, se busca la máxima eficiencia en la utilización de esos recursos.

Uno de los aspectos más innovadores de la legislación sanitaria de Catalunya fue la introducción de la diversidad de fórmulas de gestión — directa, indirecta y compartida - que puede emplear el SCS para administrar los servicios y las prestaciones del sistema. En este entorno, el SCS participó en la creación de consorcios de gestión de centros, cedió la gestión de algunos de ellos y constituyó diversas empresas públicas. También facilitó la constitución de equipos de profesionales que asumen directamente la gestión de los centros.

No obstante, a partir del análisis, de las encuestas y de las entrevistas realizadas a lo largo del estudio del SI-SSPC podemos concluir que:

La mayoría de las EP nacen del ICS cambiando la estructura estatutaria de éste por una estructura laboral para facilitar la gestión y buscar la eficiencia y eficacia en la utilización de los recursos. Las EP son proveedores del SSPC comprobándose más una vez que los cambios planteados con la aprobación de la LOSC de separación de las funciones de financiación y compra de las de provisión de servicios sanitarios no se verifican en la realidad del sector pues estas empresas son del SCS asegurador-financiador único del sistema.

Las EP no fomentan la red cooperativa, la flexibilidad y el intercambio de información en el SI-SSPC, la AATM, el IDI, el ICO, SSRCTBT, EISSA y SEMSA no mantienen relación de trabajo o de cooperación con todos los actores estudiados y las EP GSS, GPSS y el IAS están ubicados en unos entornos hospitalarios del Grupo 2 en las Regiones sanitarias de Lleida, Tarragona y Girona respectivamente.

No existe en las EP una unidad central de gestión de la innovación y de las nuevas tecnologías, tampoco existe un cargo técnico responsable de estas gestiones, por ejemplo, un Ingeniero Especialista en Organización de Empresas. Todas las demandas de inversiones en nuevas tecnologías parten de los jefes de equipos, directores o responsables de sección y no existe una coordinación general por no existir esa estructura. Esa coordinación general es hecha por los responsables de la EP de cerrar el presupuesto anual y dependiendo de los recursos económicos negociados con el SCS y con el ICS y destinados a inversiones se atiende o no las demandas.

El motor de la I+D en las EP está directamente ligado al cargo de gerente o director general y el éxito o suceso en la investigación y demás actividades 
científicas y docentes en las diferentes áreas de investigación y docencia de las EP depende directamente de ese profesional.

(8) Respecto a los hospitales del Grupo 4. Del estudio hecho se derivan, según nuestro punto de vista, las siguientes conclusiones:

Los hospitales del Grupo 4 son integrantes de la XHUP y se caracterizan por ser hospitales de alta tecnología definidos como aquellos que asumen las patologías de baja incidencia no asumibles por los demás hospitales de los Grupos 1-2 -3 de la XHUP y disponen de las llamadas supraespecialidades y de las nuevas tecnologías diagnosticoterapéuticas. Totalizan 10 hospitales, 6 ubicados en la RSBC, 1 en la RSBNM y los otros 3 en la RSCP. De los 10 hospitales 6 pertenecen al ICS [CSUVH, CSUB, ICO y HUGTiP] y los otros 4 [HC, HSJD, HSCSP y FP] son concertados por el SCS que está en negociaciones para entrar a formar parte del patronato del Clínic y del patronato del HSCSP.

No obstante, a partir del análisis, de las encuestas y de las entrevistas realizadas a lo largo del estudio del SI_SSPC podemos concluir que:

Existe un corporativismo médico muy fuerte en éstos hospitales, dificultando la gestión administrativa del propio hospital y la gestión económico financiera de la investigación científica.

Existe una investigación científica y determinados ensayos clínicos que no salen a la luz en la gestión de las fundaciones o del propio hospital —llamada investigación sumergida o investigación negra ${ }^{1}$ - , que son gestionadas por los propios médicos en convenios con laboratorios e industrias del sector, además de consumir horas de trabajo, equipamientos y fungibles del hospital como si fueran para bajas de pacientes los beneficios económicos y científicos que pudieran existir no son compartidos con la comunidad científica del centro hospitalario, pues muchas veces son de interés de la industria y laboratorios patrocinadores de esa investigación sumergida.

Estos casos de investigación sumergida son gestionados por la «mafia médica ${ }^{2}$ » que existe en estos grandes centros hospitalarios de alta tecnología perjudicando de sobremanera los intereses colectivos y la imagen de los hospitales del Grupo 4 y de

1 Estas expresiones —investigación sumergida y investigación negra — fueron aportadas al trabajo por cuatro personas entrevistadas en los hospitales de Grupo 4.

2 También esta expresión — mafia médica — fue aportada por cuatro de las personas entrevistadas en los hospitales del Grupo 4. 
la propia sociedad civil que en última instancia y a través de los impuestos proporciona los presupuestos económicos de estos centros sanitarios.

No existe en los hospitales una unidad central de gestión de la innovación y de las nuevas tecnologías, tampoco existe un cargo técnico responsable de estas gestiones, por ejemplo, un Ingeniero Especialista en Organización de Empresas. Todas las demandas de inversiones en nuevas tecnologías parten de los jefes de equipos y no existe una coordinación general por no existir esa estructura. Esa coordinación general es hecha por los responsables del hospital de cerrar el presupuesto anual y dependiendo de los recursos económicos negociados con el SCS y con el ICS y destinados a inversiones se atiende o no la demanda de los jefes de equipos.

Queda evidente la importancia del cargo «jefe de equipo» que es el motor de la $\mathrm{I}+\mathrm{D}$ en los hospitales de alta tecnología y el éxito o suceso en la investigación y demás actividades científicas y docentes en las diferentes áreas de investigación y docencia de los hospitales de alta tecnología depende directamente de ese profesional. 


\subsection{RESUMEN DE LAS CONCLUSIONES}

\begin{tabular}{|c|c|}
\hline \multicolumn{2}{|c|}{ HISTORIA HOSPITALARIA CATALANA } \\
\hline Puntos fuertes & Puntos débiles \\
\hline $\begin{array}{l}\text { - Organización de la sociedad civil } \\
\text { catalana. } \\
\text { - Sólida red de hospitales comarcales. } \\
\text { - Organizaciones con más de } 600 \\
\text { años. }\end{array}$ & $\begin{array}{l}\text { - Falta de inversiones por parte del } \\
\text { Estado en Catalunya. }\end{array}$ \\
\hline
\end{tabular}
Puntos fuertes
- Asume el mandado de la LGS.
- Mapa sanitario de Catalunya, 1981.
- Orden de acreditación, 1981.
- Creación del ICS, 1983.
- Creación de la XHUP, 1985.
- Aprobación de la LOSC, 1990.
- Creación del SCS.
- Separación de funciones de planificación-compra y provisión de servicios sanitarios.
- Reforma de la LOSC, 1995.

LOSC

Puntos débiles

- Camas de la SS representaban un $30 \%$ de toda la oferta.

- Catalunya 0,77 camas por 1000 habitantes, resto del Estado 1,91 camas por 1000 habitantes.

- La ley está desfasada en relación con la realidad del sector.

- EI SCS es a la vez planificadorcomprador y proveedor de servicios sanitarios.

\section{ENTORNO ECONÓMICO}

\section{Puntos fuertes}

- Catalunya recibirá el 5,71\% a más sobre el modelo anterior en el periodo 1998-2001.

\section{Puntos débiles}

- El sector sanitario está altamente politzado y sirve de moneda de cambio en los pactos parlamentarios de los distintos gobiernos.

\section{PLA DE SALUT}

\section{Puntos fuertes}

- Instrumento indicativo y marco de referencia para todas las actuaciones públicas en el ámbito de la salud.

- Incorpora los objetivos de salud para todo el mundo para el año 2000 de la OMS.

- Plantea 263 objetivos de salud para Catalunya en el año 2000.

- Elaborado con la participación de equipos multidisciplinarios.

- Los trabajos se inician a partir de las regiones sanitarias y de los sectores sanitarins.
Puntos débiles

- Falta de criterios en la elección de los objetivos de salud.

- Período trienal de vigencia del plan.

- No plantea el coste económico para la consecución de los objetivos de salud.

- No elaboró el calendario de las herramientas de evaluación de los objetivos de salud y tampoco el coste económico de esas acciones.

- No involucra los actores del SI en la investigación científica para la consecución de los obietivos de salud. 


\section{SCS}

Puntos fuertes

- Ente institucional que garantiza la prestación de los servicios sanitarios públicos a todos los ciudadanos.

- Planificador-evaluador-comprador de los servicios sanitarios del SSPC.

- Estructura descentralizada a través de las regiones sanitarias.

- Creación de las empresas públicas que pasan del régimen estatutario al régimen laboral.

- Creación de la Central de Balanços.

\section{Puntos débiles}

- Proveedor de servicios sanitarios al SSPC.

- No fomenta la red cooperativa dentro del SI-SSPC.

- No ha concluido la reforma de la atención primaria.

- No-creación de una Central de Resultados.

- Los informes de la Central de Balanços no son públicos.

- No existe una red de información homogénea entre los centros sanitarios públicos y concertados.

- No ha concluido la implantación de la tarjeta individual sanitaria.

\section{ICS}

\section{Puntos fuertes}

- Mayor proveedor de servicios sanitarios del SSPC.

- Gestiona centros de la Seguridad Social y de la Generalitat de Catalunya.

- Cuenta con 11 hospitales, 300 centros de atención primaria y 150 servicios de atención primaria.

\section{Puntos débiles}

- No termina de pasar por el proceso global de transformación a proveedor del sistema.

- Competencia en la provisión de servicios sanitarios con el SCS.

- No fomenta la cooperación entre sus hospitales de alta tecnología.

- No existe una red de información homogénea entre sus hospitales de alta tecnología.

\section{EMPRESAS PÚBLICAS}

\section{Puntos fuertes}

- Empresas públicas proveedoras de servicios sanitarios que pasan del régimen estatutario al régimen laboral.

- Algunas empresas públicas tienen como misión ser referencia para toda Catalunya.
Puntos débiles

- Las empresas públicas son empresas del SCS y proveedoras de servicios sanitarios al SSPC.

- No fomentan la red cooperativa dentro del SI-SSPC.

- No se relacionan con todos los actores del SI-SSPC.

- Algunas empresas públicas no tienen una misión def in ida dentro del SI-SSPC.

- No cumplen con la misión de ser referencia para toda Catalunya.

- No existe de manera formal gestión de la innovación y de las nuevas tecnologías. 


\subsection{RECOMENDACIONES}

Se presentan a continuación algunas recomendaciones sobre las políticas de fomento, que podría adoptar la Administración Pública—más particularmente el DSSS o el SCS - para lograr mejores resultados en la innovación tecnológica del SI-SSPC.

Se indica el objetivo, sin entrar detalladamente en su aplicación que en cada caso y para cada actor debe adaptarse a sus peculiaridades. En términos generales, se considera que todos los actores han de buscar el equilibrio entre los diferentes entornos, cada uno adoptando el patrón más adecuado a sus necesidades.

\section{(a) Medidas para fortalecer y orientar el entorno científico.}

- Orientación de las actividades: Se trata de fomentar las actividades orientadas a satisfacer demandas concretas del entorno social y económico en contraposición a las actividades de interés personal de los investigadores.

- Equipos multidisciplinares: Participación en las actividades de investigación de equipos multidisciplinares y elementos de distintos entornos, que aporten soluciones conjuntas para dar solución a problemas sociales y económicos.

\section{(b) Medidas para fortalecer el entorno tecnológico.}

- Desarrollo de unidades de dinamización del entorno tecnológico: En los actores donde el entorno tecnológico es apreciable, convendría dinamizar sus elementos para que contribuyan a responder a las necesidades de tecnología del entorno productivo y que aprovechen los recursos del entorno científico próximo, en buena medida la Fundaciones creadas por los hospitales de alta tecnología para gestionar la I+D tienen este objetivo.

(c) Medidas para dinamizar y mejorar la capacidad de adopción del entorno productivo

- La globalización de la economía y la rápida introducción de las nuevas tecnologías de la información y las comunicaciones en los procesos productivos y de servicios exige una adaptación periódica de los conocimientos del personal médico y técnico del SI-SSPC a las nuevas tecnologías. Sin embargo, los cursos que imparten los 
propios actores no parecen responder adecuadamente a las necesidades de los profesionales. En buena medida este objetivo esta propuesto en el documento «Catalunya en Xarxa» ya comentado anteriormente.

\section{(d) Medidas para desarrollar el entorno financiero.}

- Implantación de unidades de evaluación técnico-financiera de los contratos de prestación de servicios que tiene firmados los proveedores de servicios sanitarios con el SCS. La escasa capacidad evaluadora que tiene el SCS y las Regiones sanitarias para evaluar la calidad y eficiencia de los contratos firmados con los proveedores de servicios sanitarios se considera un freno para el desarrollo de la trasparencia, eficiencia, eficacia y calidad de esos servicios, por ello, la creación de unidades de ese tipo y la incorporación a las mismas de técnicos con formación tecnológica avanzada y con capacidad para establecer acuerdos de asesoría tecnológica con otros agentes del sistema, podría contribuir a reducir esta incertidumbre.

\subsection{LÍMITES}

Somos conscientes de los límites de nuestro estudio. Límites que afectan a dos ámbitos bien diferenciados. Por un lado, aquellos que de no haber habrían dado un mayor apoyo a las conclusiones explícitas y de otro, aquellos que tendrán que desaparecer para continuar con nuevas investigaciones.

En el primer grupo podemos incluir aquellas limitaciones que hacen referencia a la disponibilidad limitada de información. En primer lugar, el período de tiempo estudiado. La dificultad para conseguir la información o en algunos casos la inexistencia de información no ha permitido la incorporación de datos relativos a los años anteriores al período considerado.

Por otro lado, en el momento de la recogida de los datos no estaba disponible aún la información referente al año de 1998. Pero es evidente que una serie temporal más larga que la de los dos años estudiados, hubiera dado un mayor apoyo a las conclusiones obtenidas.

En segundo lugar, la identificación del Sistema de Innovación. La realidad es que el SI-SSPC no tiene un reconocimiento como entidad diferenciada en las estadísticas y estudios oficiales. Las actividades del SI son clasificadas de manera dispersa y los propios límites de aquellas resultan ambiguos. 
Por tanto, la determinación de los diferentes ámbitos del actor y de las variables estudiadas han sido hechas a partir de fuentes de información secundaria y de nuestro propio criterio a la espera de una contrastación tanto con la propia realidad innovadora de los actores como con el futuro de la investigación.

Otra limitación importante es la referente al hecho de no haber en este estudio ninguna plantilla de costes o ninguna comparación económica y financiera más profunda entre los diferentes actores y su producción científica-innovadora.

\subsection{FUTURAS INVESTIGACIONES}

El presente trabajo pretende ser una primera aproximación al estudio del Sistema de Innovación en el Sector Sanitario Público Catalán subrayando algunos de sus elementos que desde nuestro punto de vista son más significativos. El trabajo deja abierta más cuestiones de las que intenta responder, pues todavía son escasos los estudios en los que se analizan las interacciones que tienen lugar en los procesos de innovación tecnológica en el SI-SSPC. Aspectos como el establecimiento y el análisis de indicadores de interacción o la evaluación de acuerdos de cooperación, por citar solo algunos. Las futuras investigaciones que quedan abiertas tienen que estar relacionadas con la previsible evolución de esta forma organizativa. Esta evolución viene determinada por una serie de interrogantes que el SI—SSPC tiene planteados. Pensamos que esos interrogantes pueden resumirse en los siguientes:

- En primer lugar, el origen del SI—SSPC.

- En segundo lugar, el conflicto de intereses entre los diferentes actores que participan del SI-SSPC.

- En tercer lugar, la dirección o monitorización del SI-SSPC.

- En cuarto y último lugar, la inserción de las actividades del SI-SSPC en la comunidad catalana.

Dentro del ámbito estricto del desarrollo del SI_SSPC una primera cuestión de interés hace referencia a su origen y por tanto a su capacidad de reproducción y desarrollo. Esta cuestión que ha sido vista en el presente trabajo resulta de difícil solución. La mera existencia de determinados factores como son la tradición hospitalaria catalana, la existencia de instituciones seculares, los conocimientos acumulados o el creciente índice de financiación del sistema no sirve de explicación satisfactoria del origen del SI-SSPC.

Más bien se tiene que hablar de una serie de condiciones mínimas pero no suficientes a partir de las cuales los actores han aprovechado nuevas oportunidades 
que han aparecido en el sector, dentro de un contexto social y cultural determinado. En este sentido se tendría que hablar de la ausencia de factores inhibidores y no tanto de la existencia de factores determinantes de su origen. Con todo, dado su carácter idiosincrásico resulta compleja su reproducción a partir de la creación planificada de ciertas condiciones. Desde esta perspectiva las acciones posibles por parte de las instituciones competentes -importadoras y exportadoras del SI-SSPC - tendrían que tratar de eliminar los factores inhibidores o en todo caso, de coordinar y potenciar los procesos una vez iniciados hacia sistemas innovadores más que tratar de reproducir más o menos artificialmente los sistemas en nuevas localizaciones.

Un segundo problema hace referencia a la divergencia de los intereses entre los actores participantes del sistema. El funcionamiento óptimo del sistema requiere unos comportamientos determinados por parte de los actores miembros. Lo que no queda tan claro es la identificación de los intereses específicos de un actor y los del sistema. Lo que puede darse es que determinados esfuerzos individuales — publicidad, investigaciones, etc. - pueden ser apropiados parcial o totalmente por los otros actores del sistema. Determinados actores pueden ver una relación asimétrica con el sistema y optar por una protección de sus recursos específicos, cuestionando el funcionamiento básico del sistema. Así se pueden detectar posibles conflictos entre intereses y estrategias individuales y las del conjunto del sistema. La resolución de estos conflictos puede marcar el futuro del propio sistema. Si no se produce una relación correcta pude producirse una tendencia hacia el desequilibrio geográfico y total del sistema.

Otro problema que presenta el SI—SSPC es su dirección o monitorización. Hemos descrito el desarrollo de estos sistemas y su caracterización se ha mostrado eficiente en la mejora incremental y continua de los procesos, productos y servicios y para el aprovechamiento de determinadas oportunidades. Pero tenemos que preguntarnos si su reversa no será su dificultad para aprovechar aquellas oportunidades que signifiquen cambios estructurales del propio sistema. El propio funcionamiento del sistema limita la visión a largo plazo. No queda clara la capacidad del sistema para adaptarse a nuevas situaciones como son las nuevas formas de gestión compartida de la innovación, nuevas tecnologías, nuevas formas organizativas y de cooperación, red integral y compartida de información o cambios drásticos en la financiación del sistema y en las demandas de los usuarios que pueden exigir cambios organizativos y productivos profundos —envejecimiento de la población, nuevas enfermedades, disminución de la natalidad, etc-. 
Los problemas enumerados anteriormente describen un futuro complejo para el SI_-SSPC. Su resolución, desde nuestro punto de vista, puede seguir dos vías alternativas. Una sería la tendencia a la modificación y adecuación de la LOSC a la realidad del sector y la segunda pasaría por un replanteamiento del papel a jugar en el sistema por los diferentes actores.

El proceso de modificación y adecuación de la LOSC a la realidad del sector tiene un coste político elevado. Este proceso necesitaría de un acuerdo parlamentario amplio de todas las formaciones políticas con representación en el Parlament de Catalunya. Este proceso también podría crear fracturas internas dentro del sistema en relación con los actores ante la nueva estructura creada y su nueva manera de organización.

La segunda alternativa significaría un cambio o intensificación del papel de determinadas instituciones. Las instituciones públicas y concertadas que vienen jugando un papel de coordinación e incentivación de determinados esfuerzos tendrían que asumir unas mayores competencias. Se trataría de crear la red cooperativa, la gestión de la innovación y de las nuevas tecnologías para todo el sistema, la red de información estándar, homogénea y compartida y la flexibilidad de los actores hacia este nuevo modelo participativo. En definitiva se trataría de crear una agencia o centro estratégico con cierto grado de poder sobre los actores.

Las líneas futuras de investigación están implícitas en los apartados anteriores. Lo que ha quedado claro a lo largo del presente trabajo es el papel que pude desarrollar el SI-SSPC en la creación y configuración de nuevas estructuras y nuevas relaciones entre sus actores. Pero la idea a desarrollar ahora sería la de ver si el entramado de relaciones existentes y que constituyen el SI—SSPC se puede considerar como un paso intermedio a partir del cual los actores asumen un nivel de madurez y conciencia de que los factores de éxito del SI son imprescindibles para la evolución del sistema o si por el contrario tuviese que profundizarse en los factores de éxito potenciando aún más su compresión por parte de los actores del sistema.

Los aspectos tratados en este trabajo pueden constituir un punto de partida para intentar resolver la cuestión planteada. De esta manera las líneas futuras de investigación podrían plantearse en los siguientes términos:

- Aproximación a las causas explicativas de las diferencias de resultados entre los miembros del SI—SSPC de la misma actividad productiva.

En el presente trabajo se ha considerado que los actores que pertenecen al SI-SSPC tienen un cierto grado de homogeneidad. Este hecho no impide la 
existencia de diferencias entre los resultados de los actores. Tendrían que analizarse estas diferencias a partir de la estrategia de diferenciación de los actores respecto al resto de los miembros del SI-SSPC. El objetivo sería estudiar si el desarrollo a largo plazo del sistema compensa los beneficios a corto de determinadas estrategias individuales.

- Construir un marco explicativo del comportamiento de los actores de forma que pueda hacerse una evaluación de cada caso concreto.

En el presente trabajo se ha evidenciado como el desarrollo de los diversos actores en sus actividades productivas es imprescindible para el funcionamiento del sistema, pero también que cada caso concreto puede presentar un modelo diferenciado. Tendría que trabajarse en la formulación de estrategias de desarrollo del SI-SSPC para permitir su especialización y mejoría de su eficacia y eficiencia. Un tema interesante dentro de este apartado sería analizar los posibles acuerdos de cooperación entre los actores del sistema.

- Construir un marco explicativo del funcionamiento del SI-SSPC incorporando los elementos presentes en este trabajo de manera que se pueda evaluar cada caso concreto.

En el presente trabajo se ha vinculado un elemento estructural del SI-SSPC como es la producción de información por parte de sus actores. Ahora bien, la propia caracterización de los casos descritos ha permitido identificar una serie adicional de elementos: el grado de concentración geográfica, el papel jugado por los subsidios en el sistema, el corporativismo de algunas clases profesionales y, por último, las propias relaciones formales e informales entre los profesionales e instituciones del SI-SSPC. Un estudio sistemático de estos elementos puede permitir la construcción de un marco teórico explicativo del éxito o fracaso de cada caso concreto.

En definitiva consideramos que el presente trabajo supone empezar un camino para el estudio de la forma organizativa del SI en el SSPC, arrojando luz sobre algunos factores explicativos de su constitución y evolución hasta la actualidad. También, queda claro la necesidad de profundizar en el camino iniciado para obtener conclusiones que den solución a las numerosas cuestiones aún no resueltas y que el propio trabajo nos ha aportado. 
